\title{
Evaluation of Communication Command Effectiveness Based on Fuzzy Analytic Hierarchy Process \\ CAO Weixi ${ }^{1, a}$, ZHU Jisheng $^{1}$, REN Xuejun $^{1}$ \\ ${ }^{1}$ Engineering university of the Chinese people's armed police force, Xi'an 710086, China \\ a453962509@qq.com
}

Keywords: Fuzzy AHP; Communication Command Efficiency; Evaluation

\begin{abstract}
Forces carries out the task to improve effectiveness of communication command, is the important guarantee of communication support ability to ascend, the fuzzy analytic hierarchy process (AHP) to evaluate communication command efficiency, to a certain degree of communication security work guide role. This paper established the hierarchy communication command effectiveness evaluation index system. Combine and fuzzy comprehensive evaluation and analytic hierarchy process (AHP), this paper proposes a communication command effectiveness evaluation model based on fuzzy analytic hierarchy process (AHP), according to the characteristics of communication command effectiveness evaluation index and fuzzy complex assessment and objective assessment results are drawn.
\end{abstract}

\section{Introduction}

Construction and utilization of information system have profound influence on army combat mode. Traditional empirical command which cannot meet the demand of information warfare is replaced with intelligent command mode supported by information technology means. Communication command effectiveness can only be comprehensively improved by relying on information system, giving full play to functional advantages of information system in communication command, realizing real-time, integrated and transparent command, and improving command decision-making to be more scientific. However, there are still some problems in communication command through information system platform, and these problems restrict the full play of command effectiveness. Therefore, scientific evaluation methods shall be used in quantitative evaluation of communication command effectiveness based on information system, in order to provide a basis for improving communication command effectiveness through scientific application of information system.

\section{Application of AHP in evaluation of communication command effectiveness}

\section{Establish an evaluation index system}

Communication command effectiveness relies on organic operation of multiple factors and the overall effectiveness can be affected by situational awareness, command and control capability, and communication support capability etc in mission area. This is the target layer. A four-level index system can be obtained by decomposing communication command effectiveness model and the general target of communication command effectiveness layer by layer based on information system according to analytic hierarchy process (AHP), and the index system structure is shown in figure 1 . 


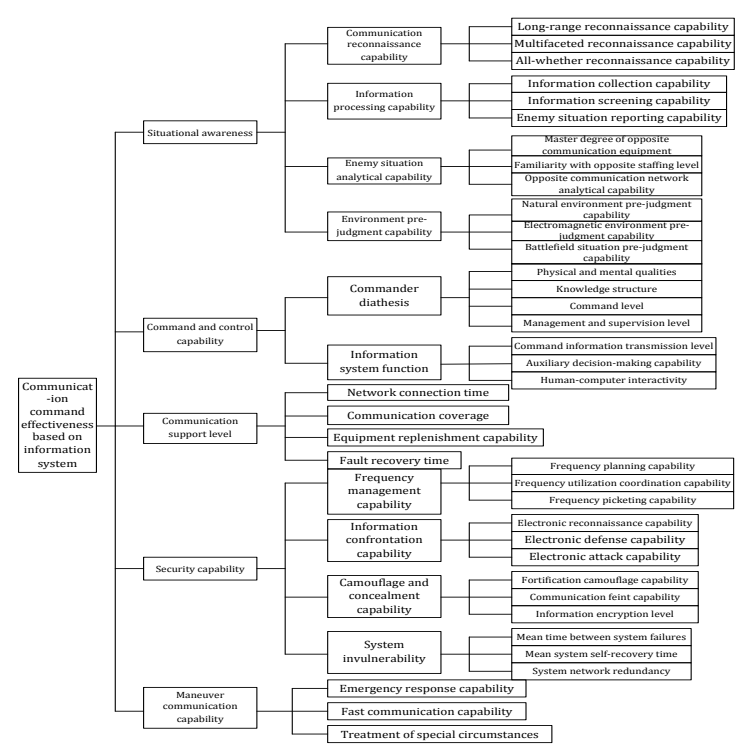

Fig. 1. Evaluation index system for communication command effectiveness based on information system

\section{Construct judgment matrix for each level of indexes}

Establish factor sets of each level, compare between two factors and assign values according to T. L. Satty's 1-9 scale method to denote importance degrees to each other, as shown in table 1.

Table 1 Index weight scale by AHP

\begin{tabular}{|c|c|}
\hline Valuec & Meaning \\
\hline 1 & Index $i$ is equally important with index $\mathrm{j}$ \\
3 & Index $\mathrm{i}$ is slightly more important than index $\mathrm{j}$ \\
5 & Index $\mathrm{i}$ is obviously more important than index $\mathrm{j}$ \\
9 & Index $\mathrm{i}$ is certainly more important than index $\mathrm{j}$ \\
\hline & Index $\mathrm{i}$ is absolutely more important than index $\mathrm{j}$ \\
\hline $2,4,6$ and 8 are other available medians; if index $\mathrm{i}$ is less important than index $\mathrm{j}$, the value is $1 / \mathrm{v}$, where $\mathrm{v}$ is $1-9$ \\
\hline
\end{tabular}

\section{Calculate weight vectors of matrix}

In AHP, characteristic roots method is the most widely used in weight calculation. Suppose judgment matrix $\mathrm{A}$ and equation

$$
\mathbf{A w}=\lambda_{\text {max }} \mathbf{w}
$$

Where $\lambda_{\max }$ is the maximum characteristic root of judgment matrix $\mathrm{A}$, and can be the required weight vector after normalization processing by vector $\mathrm{W}$ obtained by calculating the equation.

\section{Consistency test of judgment matrix}

To avoid illogical misjudgment, consistency of judgment matrix A shall be tested:

Firstly, calculate C.I. (consistency index) of the matrix

$$
\text { C.I. }=\frac{\lambda_{\max }-n}{n-1}
$$

Then, look up average random consistency index R.I. (random index) corresponding to the matrix. See details in table 2 .

Table 2 R.I. of $1 \sim 15$-order positive reciprocal matrix R.I.

\begin{tabular}{|c|ccccccc|}
\hline Matrix order & 1 & 2 & 3 & 4 & 5 & 6 & 7 \\
\hline R.L & 0 & 0 & 0.52 & 0.89 & 1.12 & 1.26 & 1.36 \\
\hline Matrix order & 8 & 9 & 10 & 11 & 12 & 13 & 14 \\
\hline R.L & 1.41 & 1.46 & 1.49 & 1.52 & 1.54 & 1.56 & 1.58 \\
\hline
\end{tabular}

Calculate C.R.(consistency ratio)

$$
C . R .=\frac{C . I .}{R . I .}
$$

When C.R. $<0.1$, the consistency of judgment matrix $\mathrm{A}$ is feasible; and when C.R. $\geq 0.1$, judgment matrix A shall be corrected appropriately. 
Table of comparison of relative importance among all levels of indexes and their weights

Table 3 Table of comparison of relative importance among second-level indexes and their weights

\begin{tabular}{|c|c|c|c|c|c|c|}
\hline Score ${ }^{\text {Index }}$ & $\begin{array}{l}\text { Situational } \\
\text { awarness }\end{array}$ & $\begin{array}{l}\text { Command and } \\
\text { control capability }\end{array}$ & $\begin{array}{l}\text { Communication } \\
\text { support level }\end{array}$ & $\begin{array}{l}\text { Security } \\
\text { capability }\end{array}$ & $\begin{array}{c}\text { Maneuver } \\
\text { communication } \\
\text { capability }\end{array}$ & Weigh \\
\hline Situational awareness & 1 & 1 & 2 & 1 & $1 / 2$ & 0.1992 \\
\hline $\begin{array}{c}\text { Command and control } \\
\text { capability }\end{array}$ & 1 & 1 & 1 & 2 & 1 & 0.2289 \\
\hline $\begin{array}{c}\text { Communication support } \\
\text { level }\end{array}$ & $1 / 2$ & 1 & 1 & 1 & 2 & 0.1992 \\
\hline Security capability & 1 & $1 / 2$ & 1 & 1 & 1 & 0.1734 \\
\hline $\begin{array}{l}\text { Mineuver communication } \\
\text { capability }\end{array}$ & 2 & 1 & $1 / 2$ & 1 & 1 & 0.1992 \\
\hline
\end{tabular}

Note: consistency test result is 0.0800 , the maximum characteristic root is 5.3582 , and the overall target weight is 1 .

Table 4 Table of comparison of relative importance among third-level indexes under situational awareness index and their weights

\begin{tabular}{|c|c|c|c|c|c|}
\hline Index & $\begin{array}{c}\text { Communication } \\
\text { reconnaissance capability }\end{array}$ & $\begin{array}{c}\text { Information processing } \\
\text { capability }\end{array}$ & $\begin{array}{c}\text { Enemy situation } \\
\text { analytical capability }\end{array}$ & $\begin{array}{c}\text { Environment pre- } \\
\text { judgment capability }\end{array}$ & Weight \\
\hline $\begin{array}{c}\text { Communication } \\
\text { reconnaissance capability }\end{array}$ & 1 & $1 / 3$ & $1 / 3$ & $1 / 2$ & 0.1045 \\
\hline $\begin{array}{c}\text { Information processing } \\
\text { capability }\end{array}$ & 3 & 1 & 2 & 4 & 0.4764 \\
\hline $\begin{array}{c}\text { Enemy situation analytical } \\
\text { capability }\end{array}$ & $1 / 2$ & 3 & 1 & 1 & 0.2382 \\
\hline $\begin{array}{c}\text { Environment pre-judgment } \\
\text { capability }\end{array}$ & $1 / 4$ & 2 & 1 & 1 & 0.1810 \\
\hline
\end{tabular}

Note: consistency test result is 0.04946 , the maximum characteristic root is 4.132 , and the overall target weight is 0.1992 .

Table 5 Table of comparison of relative importance among third-level indexes under command and control capability index and their weights

\begin{tabular}{|c|c|c|c|}
\hline Index & Commander diathesis & Information system function & Weight \\
\hline Commander diathesis & 1 & $1 / 2$ & 0.3333 \\
\hline Information system function & 2 & 1 & 0.6667 \\
\hline
\end{tabular}

Note: consistency test result is 0.0000 , the maximum characteristic root is 2.0000 , and the overall target weight is 0.2289 .

Table 6 Table of comparison of relative importance among third-level indexes under communication support capability index and their weights

\begin{tabular}{|c|c|c|c|c|c|}
\hline Index & $\begin{array}{c}\text { Network } \\
\text { connection time }\end{array}$ & $\begin{array}{c}\text { Communication } \\
\text { coverage }\end{array}$ & $\begin{array}{c}\text { Equipment replenishment } \\
\text { capability }\end{array}$ & $\begin{array}{c}\text { Fault recovery } \\
\text { time }\end{array}$ & Weight \\
\hline Network connection time & 1 & 2 & $1 / 3$ & 3 & 0.2470 \\
\hline Communication coverage & $1 / 2$ & 1 & $1 / 3$ & $1 / 2$ & 0.1116 \\
\hline $\begin{array}{c}\text { Equipment replenishment } \\
\text { capability }\end{array}$ & 3 & 3 & 1 & 4 & 0.5087 \\
\hline Fault recovery time & $1 / 3$ & 2 & $1 / 4$ & 1 & 0.1327 \\
\hline
\end{tabular}

Note: consistency test result is 0.0788 , the maximum characteristic root is 4.2104 , and the overall target weight is 0.1992 .

Table 7 Table of comparison of relative importance among third-level indexes under security capability index and their weights

\begin{tabular}{|c|c|c|c|c|c|}
\hline Index & $\begin{array}{c}\text { Frequency management } \\
\text { capability }\end{array}$ & $\begin{array}{c}\text { Information } \\
\text { confrontation capability }\end{array}$ & $\begin{array}{c}\text { Camouflage and } \\
\text { concealment capability }\end{array}$ & $\begin{array}{c}\text { System } \\
\text { invulnerability }\end{array}$ & Weight \\
\hline $\begin{array}{c}\text { Frequency manasgement } \\
\text { cappability }\end{array}$ & 1 & 4 & 2 & 5 & 0.5101 \\
\hline $\begin{array}{c}\text { Information confrontation } \\
\text { cappability }\end{array}$ & $1 / 4$ & 1 & $1 / 3$ & 1 & 0.1091 \\
\hline $\begin{array}{c}\text { Cammonflage and } \\
\text { concealment capability }\end{array}$ & $1 / 2$ & 3 & 1 & 2 & 0.2669 \\
\hline System invulnerability & $1 / 5$ & 1 & $1 / 2$ & 1 & 0.1141 \\
\hline
\end{tabular}

Note: consistency test result is 0.0101 , the maximum characteristic root is 4.0268 , and the overall target weight is 0.1743 . 
Table 8 Table of comparison of relative importance among third-level indexes under maneuver communication capability index and their weights

\begin{tabular}{|c|c|c|c|c|}
\hline Index & $\begin{array}{c}\text { Emergency response } \\
\text { capability }\end{array}$ & $\begin{array}{c}\text { Fast communication } \\
\text { capability }\end{array}$ & $\begin{array}{c}\text { Treatment of special } \\
\text { circumstances }\end{array}$ & Weight \\
\hline Emergency response capability & 1 & 2 & 3 & 0.5499 \\
\hline Fast communication capability & $1 / 2$ & 1 & 1 & 0.2402 \\
\hline $\begin{array}{c}\text { Treatment of special } \\
\text { circumstances }\end{array}$ & $1 / 3$ & 1 & 1 & 0.2098 \\
\hline
\end{tabular}

Note: consistency test result is 0.0036, the maximum characteristic root is 3.0037 , and the overall target weight is 0.1992 .

Table 9 Table of comparison of relative importance among fourth-level indexes under communication reconnaissance capability index and their weights

\begin{tabular}{|c|c|c|c|c|}
\hline Index & $\begin{array}{c}\text { Long-range } \\
\text { reconnaissance capability }\end{array}$ & $\begin{array}{c}\text { Multifaceted reconnaissance } \\
\text { capability }\end{array}$ & $\begin{array}{c}\text { All-whether } \\
\text { reconnaissancecapability }\end{array}$ & Weight \\
\hline $\begin{array}{c}\text { Long-range } \\
\text { reconnaissance capability }\end{array}$ & 1 & 2 & 3 & 0.5499 \\
\hline $\begin{array}{c}\text { Multifaceted } \\
\text { reconnaissance capability }\end{array}$ & $1 / 2$ & 1 & 1 & 0.2402 \\
\hline $\begin{array}{c}\text { All-whether } \\
\text { reconnaissance capability }\end{array}$ & $1 / 3$ & 1 & 1 & 0.2098 \\
\hline
\end{tabular}

Table 10 Table of comparison of relative importance among fourth-level indexes under information processing capability index and their weights

\begin{tabular}{|c|c|c|c|c|}
\hline Index & $\begin{array}{c}\text { Information collection } \\
\text { capability }\end{array}$ & $\begin{array}{c}\text { Information screening } \\
\text { capability }\end{array}$ & $\begin{array}{c}\text { Enemy situation } \\
\text { reporting capability }\end{array}$ & Weight \\
\hline $\begin{array}{c}\text { Information collection } \\
\text { capability }\end{array}$ & 1 & 3 & 5 & 0.6370 \\
\hline $\begin{array}{c}\text { Information screening } \\
\text { capability }\end{array}$ & $1 / 3$ & 1 & 3 & 0.2583 \\
\hline $\begin{array}{c}\text { Enemy situation } \\
\text { reporting capability }\end{array}$ & $1 / 3$ & $1 / 5$ & 1 & 0.1047 \\
\hline
\end{tabular}

Note: consistency test result is 0.0370 , the maximum characteristic root is 4.0000 , and the overall target weight is 0.0949 .

Table 11 Table of comparison of relative importance among fourth-level indexes under enemy situation analytical capability index and their weights

\begin{tabular}{|c|c|c|c|c|}
\hline Index & $\begin{array}{c}\text { Master degroc of opposite } \\
\text { communication equipment }\end{array}$ & $\begin{array}{c}\text { Familiarity with } \\
\text { opposite staffing level }\end{array}$ & $\begin{array}{c}\text { Opposite communication } \\
\text { network analytical capability }\end{array}$ & Weight \\
\hline $\begin{array}{c}\text { Master degree of opposite } \\
\text { communication equipment }\end{array}$ & 1 & $1 / 3$ & $1 / 5$ & 0.1095 \\
\hline $\begin{array}{c}\text { Familiarity with opposite } \\
\text { staffing level }\end{array}$ & 3 & 1 & $1 / 2$ & 0.1090 \\
\hline $\begin{array}{c}\text { Opposite communication } \\
\text { network analytical capability }\end{array}$ & 5 & 2 & 1 & 0.5816 \\
\hline
\end{tabular}

Note: consistency test result is 0.0036 , the maximum characteristic root is 3.0037 , and the overall target weight is 0.0475 .

Table 12 Table of comparison of relative importance among fourth-level indexes under environment pre-judgment capability index and their weights

\begin{tabular}{|c|c|c|c|c|}
\hline Index & $\begin{array}{l}\text { Natural environment pre- } \\
\text { judgment capnatility }\end{array}$ & $\begin{array}{l}\text { Electromagnetic environment } \\
\text { pre-jutgment capubility }\end{array}$ & $\begin{array}{l}\text { Battleficld situation pre- } \\
\text { judgment cappability }\end{array}$ & wcight \\
\hline $\begin{array}{l}\text { Natural cavironment pre-judgment } \\
\text { capability }\end{array}$ & 1 & $1 / 2$ & 2 & 0.2857 \\
\hline $\begin{array}{l}\text { Filoctromagnetic cavironment pre- } \\
\text { judpment alpability }\end{array}$ & 2 & 1 & 4 & 0.5714 \\
\hline $\begin{array}{l}\text { Batllefield situation pre-judgment } \\
\text { capability }\end{array}$ & $1 / 2$ & $1 / 4$ & 1 & 0.1429 \\
\hline
\end{tabular}

Note: consistency test result is 0.0000 , the maximum characteristic root is 3.0000 , and the overall target weight is 0.0361 . 
Table 13 Table of comparison of relative importance among fourth-level indexes under commander diathesis index and their weights

\begin{tabular}{|c|c|c|c|c|c|}
\hline Index & $\begin{array}{c}\text { Physical and } \\
\text { mental qualities }\end{array}$ & $\begin{array}{c}\text { Knowledge } \\
\text { structure }\end{array}$ & Command level & $\begin{array}{c}\text { Managenent and } \\
\text { supervision level }\end{array}$ & Weight \\
\hline $\begin{array}{c}\text { Physical and mental } \\
\text { qualities }\end{array}$ & 1 & $1 / 5$ & $1 / 3$ & 2 & 0.1259 \\
\hline Knowledge structure & 5 & 1 & 2 & 3 & 0.4878 \\
\hline Command level & 3 & $1 / 2$ & 1 & 2 & 0.2743 \\
\hline $\begin{array}{c}\text { Management and } \\
\text { supervision level }\end{array}$ & $1 / 2$ & $1 / 3$ & $1 / 2$ & 1 & 0.1120 \\
\hline
\end{tabular}

Note: consistency test result is 0.0660 , the maximum characteristic root is 4.1763 , and the overall target weight is 0.0763 .

Table 14 Table of comparison of relative importance among fourth-level indexes under information system function index and their weights

\begin{tabular}{|c|c|c|c|c|}
\hline Index & $\begin{array}{c}\text { Command information } \\
\text { transmission level }\end{array}$ & $\begin{array}{c}\text { Auxiliary decision- } \\
\text { making capability }\end{array}$ & $\begin{array}{c}\text { Human-computcr } \\
\text { intcractivity }\end{array}$ & Weight \\
\hline $\begin{array}{c}\text { Command information } \\
\text { transmission level }\end{array}$ & 1 & $1 / 2$ & $1 / 3$ & 0.1574 \\
\hline $\begin{array}{c}\text { Auxiliary decision-making } \\
\text { capability }\end{array}$ & 2 & 1 & $1 / 3$ & 0.2493 \\
\hline Human computer interactivity & 3 & 3 & 1 & 0.5936 \\
\hline
\end{tabular}

Note: consistency test result is 0.0516 , the maximum characteristic root is 3.0536 , and the overall target weight is 0.1526 .

Table 15 Table of comparison of relative importance among fourth-level indexes under frequency management capability index and their weights

\begin{tabular}{|c|c|c|c|c|}
\hline Index & $\begin{array}{c}\text { Frequency planning } \\
\text { capability }\end{array}$ & $\begin{array}{c}\text { Frequency utilization } \\
\text { coordination capability }\end{array}$ & $\begin{array}{c}\text { Frequency picketing } \\
\text { capability }\end{array}$ & Wcight \\
\hline $\begin{array}{c}\text { Frequency planning } \\
\text { capability }\end{array}$ & 1 & 3 & 1 & 0.6114 \\
\hline $\begin{array}{c}\text { Frequency utilization } \\
\text { coordination capability }\end{array}$ & $1 / 3$ & 1 & 3 & $\mathbf{0 . 6 2 8 4}$ \\
\hline $\begin{array}{c}\text { Frequency picketing } \\
\text { capability }\end{array}$ & $1 / 4$ & $1 / 3$ & 1 & 0.1172 \\
\hline
\end{tabular}

Note: consistency test result is 0.0707 , the maximum characteristic root is 3.0735 , and the overall target weight is 0.0885 .

Table 16 Table of comparison of relative importance among fourth-level indexes under information confrontation capability index and their weights

\begin{tabular}{|c|c|c|c|c|}
\hline Index & $\begin{array}{c}\text { Electronic reconnaissance } \\
\text { capability }\end{array}$ & $\begin{array}{c}\text { Electronic defense } \\
\text { capability }\end{array}$ & $\begin{array}{c}\text { Electronic attack } \\
\text { capability }\end{array}$ & Weight \\
\hline Electronic reconnaissance capability & 1 & 2 & $1 / 2$ & 0.2970 \\
\hline Electronic defense capability & $1 / 2$ & 1 & $1 / 3$ & 0.1634 \\
\hline Electronic attack capability & 2 & 3 & 1 & 0.5396 \\
\hline
\end{tabular}

Note: consistency test result is 0.0088 , the maximum characteristic root is 3.0092, and the overall target weight is 0.0189 .

Table 17 Table of comparison of relative importance among fourth-level indexes under camouflage and concealment capability index and their weights

\begin{tabular}{|c|c|c|c|c|}
\hline Index & $\begin{array}{c}\text { Fortification camouflage } \\
\text { capability }\end{array}$ & $\begin{array}{c}\text { Communication feint } \\
\text { capability }\end{array}$ & $\begin{array}{c}\text { Information encryption } \\
\text { level }\end{array}$ & Weight \\
\hline Fortification camouflage capability & 1 & 3 & 7 & 0.6586 \\
\hline Communication feint capability & $1 / 3$ & 1 & 4 & 0.2628 \\
\hline Information encryption level & $1 / 7$ & $1 / 4$ & 1 & 0.0786 \\
\hline
\end{tabular}

Note: consistency test result is 0.0311 , the maximum characteristic root is 3.0324 , and the overall target weight is 0.0463 . 
Table 18 Table of comparison of relative importance among fourth-level indexes under system invulnerability index and their weights

\begin{tabular}{|c|c|c|c|c|}
\hline Index & $\begin{array}{c}\text { Mean time between } \\
\text { system failures }\end{array}$ & $\begin{array}{c}\text { Mean system self- } \\
\text { recovery time }\end{array}$ & $\begin{array}{c}\text { System network } \\
\text { redundancy }\end{array}$ & Weight \\
\hline Mean time between system failures & 1 & 2 & 4 & 0.5584 \\
\hline Mean system self-recovery time & $1 / 2$ & 1 & 3 & 0.3169 \\
\hline System network redundancy & $1 / 4$ & $1 / 3$ & 1 & 0.1220 \\
\hline
\end{tabular}

Note: consistency test result is 0.0176 , the maximum characteristic root is 3.0183 , and the overall target weight is 0.0198 .

Consistency test results of the above judgment matrixes are smaller than 0.1 , and thus these matrixes have passed the test.

\section{Evaluation of communication support effectiveness in joint operations by fuzzy comprehensive evaluation method}

\section{Establish evaluation standards}

Set ranges of all grades of variable values as: $0-0.4$ (poor), $0.4-0.6$ (general), $0.6-0.8$ (good), and $0.8-1.0$ (excellent). Thus, evaluation grade matrix is:

$$
P=\left(\begin{array}{l}
0.9 \\
0.7 \\
0.5 \\
0.2
\end{array}\right)
$$

\section{Calculate membership values}

Conduct normalization processing of expert comments, and calculate membership of evaluation index sets, as shown in table 19.

Table 19 Table of membership of evaluation index set

\begin{tabular}{|c|c|c|c|c|}
\hline $\begin{array}{ll} & \text { Comment } \\
\text { Membership } & \end{array}$ & Excellent & Good & General & Poor \\
\hline Long-range reconnaissance capability & 0.1458 & 0.5833 & 0.2083 & 0.0625 \\
\hline Multifaceted reconnaissance capability & 0.1875 & 0.6458 & 0.1250 & 0.0417 \\
\hline All-whether reconnaissance capability & 0.3333 & 0.5208 & 0.0417 & $\overline{0.1042}$ \\
\hline Information collection capability & 0.2080 & 0.1458 & 0.7917 & 0.0417 \\
\hline Information screening capability & 0.0625 & 0.1042 & 0.3958 & 0.4375 \\
\hline Enemy situation reporting capability & 0.0417 & 0.0625 & 0.5417 & 0.3542 \\
\hline Master degree of opposite communication equipment & 0.3333 & 0.6250 & 0.0208 & 0.0208 \\
\hline Familiarity with opposite staffing level & 0.2083 & 0.6042 & 0.0208 & 0.1667 \\
\hline Opposite communication network analytical capability & 0.1667 & 0.5625 & 0.1667 & 0.1042 \\
\hline Natural environment pre-judgment capability & 0.1458 & 0.5833 & 0.1667 & 0.1042 \\
\hline Electromagnetic environment pre-judgment capability & 0.1857 & 0.5625 & 0.1875 & 0.0625 \\
\hline Battlefield situation pre-judgment capability & 0.2292 & 0.6250 & 0.1458 & 0.0000 \\
\hline $\begin{array}{l}\text { Physical and mental qualities } \\
\end{array}$ & 0.2083 & 0.6458 & 0.1042 & 0.0417 \\
\hline Knowledge structure & 0.1042 & 0.5000 & 0.2292 & 0.1667 \\
\hline Command level & 0.0625 & 0.6042 & 0.3125 & 0.0208 \\
\hline Management and supervision level & 0.1667 & 0.5625 & 0.1875 & 0.0833 \\
\hline Command information transmission level & 0.1875 & 0.1458 & 0.5208 & 0.1458 \\
\hline Auxiliary decision-making capability & 0.0417 & 0.1875 & 0.6042 & 0.1667 \\
\hline Human-computer interactivity & 0.2708 & 0.3958 & 0.3125 & 0.0208 \\
\hline Network connection time & 0.2500 & 0.5625 & 0.1250 & 0.0625 \\
\hline Communication coverage & 0.0625 & 0.5833 & 0.2500 & 0.1042 \\
\hline Equipment replenishment capability & 0.4583 & 0.5208 & 0.0208 & 0.0000 \\
\hline Fault recovery time & 0.3750 & 0.5417 & 0.0208 & 0.0625 \\
\hline Frequency planning capability & 0.3333 & 0.5625 & 0.0625 & 0.0417 \\
\hline Frequency utilization coordination capability & 0.4792 & 0.4583 & 0.0417 & 0.0208 \\
\hline Frequency picketing capability & 0.4375 & 0.5000 & 0.0208 & 0.0417 \\
\hline Electronic reconnaissance capability & 0.2292 & 0.6042 & 0.0833 & 0.0833 \\
\hline Electronic defense capability & 0.1667 & 0.5208 & 0.2708 & 0.0417 \\
\hline Electronic attack capability & 0.3333 & 0.6250 & 0.0417 & 0.0000 \\
\hline Fortification camouflage capability & 0.1667 & 0.5417 & 0.1875 & 0.1042 \\
\hline Communication feint capability & 0.2500 & 0.5625 & 0.0625 & 0.1250 \\
\hline Information encryption level & 0.1875 & 0.5208 & 0.1458 & 0.1458 \\
\hline Mean time between system failures & 0.2708 & 0.5417 & 0.0000 & 0.1875 \\
\hline Mean system self-recovery time & 0.2917 & 0.5833 & 0.0833 & 0.0417 \\
\hline System network redundancy & 0.1042 & 0.5417 & 0.1875 & 0.1667 \\
\hline Emergency response capability & 0.3542 & 0.5000 & 0.1250 & 0.0208 \\
\hline Fast communication capability & 0.3125 & 0.4375 & 0.1667 & 0.0833 \\
\hline Treatment of special circumstances & 0.1875 & 0.6042 & 0.1875 & 0.0208 \\
\hline
\end{tabular}




\section{Establish fuzzy evaluation matrixes}

Denote row vector of matrix $\mathrm{R}$ as $R_{i}$, element $R_{i j}$ of which denotes membership of index to grade $\mathrm{j}$, and matrix $A_{i}$ is the weight of this level of indexes. Calculate value set of fuzzy comprehensive membership.

$$
\begin{aligned}
& R_{11}=\left(\begin{array}{l}
R_{111} \\
R_{112} \\
R_{113}
\end{array}\right)=\left(\begin{array}{llll}
0.1458 & 0.5833 & 0.2083 & 0.0625 \\
0.1875 & 0.6458 & 0.1250 & 0.0417 \\
0.3333 & 0.5208 & 0.0417 & 0.1042
\end{array}\right) \\
& B_{11}=A_{11} \bullet R_{11}=\left(\begin{array}{l}
0.5499 \\
0.2402 \\
0.2098
\end{array}\right)^{T} \bullet R_{11}=\left(\begin{array}{llll}
0.1951 & 0.5851 & 0.1533 & 0.066
\end{array}\right) \\
& B_{12}=A_{12} \bullet R_{12}=\left(\begin{array}{l}
0.6370 \\
0.2583 \\
0.1047
\end{array}\right)^{T} \bullet\left(\begin{array}{llll}
0.2080 & 0.1458 & 0.7917 & 0.0417 \\
0.0625 & 0.1042 & 0.3958 & 0.4375 \\
0.0417 & 0.0625 & 0.5417 & 0.3542
\end{array}\right) \\
& =\left(\begin{array}{llll}
0.1530 & 0.1263 & 0.6632 & 0.1766
\end{array}\right) \\
& B_{13}=A_{13} \bullet R_{13}=\left(\begin{array}{l}
0.1095 \\
0.1090 \\
0.5816
\end{array}\right)^{T} \bullet\left(\begin{array}{llll}
0.3333 & 0.6250 & 0.0208 & 0.0208 \\
0.2083 & 0.6042 & 0.0208 & 0.1667 \\
0.1667 & 0.5625 & 0.1667 & 0.1042
\end{array}\right) \\
& =\left(\begin{array}{llll}
0.1561 & 0.4614 & 0.1014 & 0.0810
\end{array}\right) \\
& B_{14}=A_{14} \bullet R_{14}=\left(\begin{array}{l}
0.2857 \\
0.5714 \\
0.1429
\end{array}\right)^{T} \bullet\left(\begin{array}{llll}
0.1458 & 0.5833 & 0.1667 & 0.1042 \\
0.1857 & 0.5625 & 0.1875 & 0.0625 \\
0.2292 & 0.6250 & 0.1458 & 0.0000
\end{array}\right) \\
& =\left(\begin{array}{llll}
0.1805 & 0.5773 & 0.1755 & 0.0654
\end{array}\right) \\
& B_{1}=A_{1} \bullet R_{1}=\left(\begin{array}{l}
0.1045 \\
0.4764 \\
0.2382 \\
0.1810
\end{array}\right)^{T} \bullet\left(\begin{array}{llll}
0.1951 & 0.5851 & 0.1533 & 0.0665 \\
0.1530 & 0.1263 & 0.6632 & 0.1766 \\
0.1561 & 0.4614 & 0.1014 & 0.0810 \\
0.1805 & 0.5773 & 0.1755 & 0.0654
\end{array}\right) \\
& =\left(\begin{array}{llll}
0.1631 & 0.3357 & 0.3879 & 0.1222
\end{array}\right)
\end{aligned}
$$

Similarly, fuzzy comprehensive evaluation result of overall target can be obtained as follows:

$$
B=\left(\begin{array}{llll}
0.2717 & 0.4731 & 0.2182 & 0.0704
\end{array}\right)
$$

Thus, comprehensive evaluation value is:

$$
Q=B \bullet P=\left(b_{1}, b_{2}, \cdots, b_{m}\right) \bullet\left(\begin{array}{l}
0.9 \\
0.7 \\
0.5 \\
0.2
\end{array}\right)=0.6989
$$

Variable range of value $\mathrm{Q}$ is corresponding to grade $0.6-0.8$ (good), which is the final evaluation result.

Through fuzzy calculation, the evaluation value of communication command effectiveness based on information system is 0.6989 , and the evaluation result is "good", which is basically in line with reality. 


\section{Conclusions}

With information system, communication command effectiveness is significantly improved in army task execution and communication support capability is also substantially strengthened. However, with constant deepening of information construction, the key of being initiative in wars lies in high integration of information system utilization with communication command, so that army communication command mechanism can better adapt to application of information achievements and communication command effectiveness based on information system can be comprehensively improved.

\section{References}

[1] Saaty TL. Modeling unstructured decision problems-the theory of analytical hierarchies [J]. Math Comput Simulation, 1978 20: 147-158.

[2] QU Tian-bao. Introduction to communication ability [ $\mathrm{M}]$. Beijing: PLA Publishing House,1999.

[3] Xiong Li,Liang Liang,Wang Guo-hua. Method research on selection and valuation of numeric scale in analytic hierarchy process $[\mathrm{J}]$. Systems Engineering-theory \& Practice,2005 25( 3) : $72-$ 79.

[4] Li Yong,Hu Xiang-hong,Qiao Jian. An improved fuzzy AHP method [ J ] . Journal of Northwest University( NaturalScience Edition),2005 35( 1) : 11 - 12.

[5] Zhang Ji-jun. Fuzzy analytical hierarchy process [J] . Fuzzy Systems and Mathematics, 2000 14( 2) : $80-88$. 